\title{
Serotonin mediates a learned increase in attraction to high concentrations of benzaldehyde in aged C. elegans
}

\author{
David Tsui ${ }^{1}$ and Derek van der Kooy \\ Department of Medical Genetics and Microbiology, University of Toronto, Toronto, Ontario M5S 3E1, Canada
}

\begin{abstract}
We utilized olfactory-mediated chemotaxis in Caenorhabditis elegans to examine the effect of aging on information processing and animal behavior. Wild-type (N2) young adults (day 4) initially approach and eventually avoid a point source of benzaldehyde. Aged adult animals (day 7) showed a stronger initial approach and a delayed avoidance to benzaldehyde compared with young adults. This delayed avoidance is due to an increased attraction rather than a decreased avoidance to benzaldehyde because (1) aged odr-3 mutants that are defective in odor attraction showed no delayed benzaldehyde avoidance, and (2) the delay in avoidance was also observed with another attractant diacetyl, but not the repellent octanol. Interestingly, the stronger expression of attractive behavior was only observed at benzaldehyde concentrations of $1 \%$ or higher. When worms were grown on nonbacterial growth media instead of Escherichia coli, thus removing the contingency between odors released from the food and the food itself, the increase in attraction to benzaldehyde disappeared. The increased attraction recovered after reinitiating the odor-food contingency by returning animals to $E$. coli food or supplementing axenic media with benzaldehyde. Moreover, serotonin-deficient mutants showed a deficit in the age-enhanced attraction. These results suggest that the increased attraction to benzaldehyde in aged worms is (1) serotonin mediated, (2) specific to high concentration of odorants, and (3) dependent on a learned association of odor metabolites with the presence of food. We propose that associative learning may selectively modify pathways at or downstream from a low-affinity olfactory receptor.
\end{abstract}

Aging is a process of gradual deterioration of physiological function with increase in age. Caenorhabditis elegans exhibit functional declines that are analogous to humans, such as loss of muscle mass and function (Herndon et al. 2002; Evason et al. 2005), making it a good model for the study of different aspects of aging in specific tissues. Moreover, because of its powerful genetic database and a broad range of genetic techniques, C. elegans is widely used as the model for studying the genetics of aging. While most studies of aging in C. elegans focus on easily observable traits such as life span and lipofuscin accumulation (Boehm and Slack 2005; Gerstbrein et al. 2005), few studies have looked at the behavioral consequences of $C$. elegans aging.

One of the behavioral changes that occur with aging is a change in sensory function. In humans, the ability to identify smells peaks between the third and fifth decades of life and declines to the end of life (Doty et al. 1984). Olfactory-mediated chemotaxis in C. elegans requires the integration of sensory and motor function and provides a relatively simple system to study how aging affects olfactory-mediated behaviors. C. elegans can detect and approach or avoid many odorants (Bargmann et al. 1993; Troemel et al. 1995). One of these odorants, benzaldehyde, is sensed by the AWC primary chemosensory neuron (Bargmann et al. 1993) and is attractive at low concentrations. At high benzaldehyde concentration, animals show a biphasic behavior in which they initially approach the odorant and then show strong aversion to the odorant (Nuttley et al. 2001). Two previous studies have examined the effect of age on olfactory-mediated responses. An early study showed that aged C. elegans demonstrates reduced movement toward Escherichia coli, the worm's food (Hosono 1978). Using chemotaxis and motor locomotion assays, a

${ }^{1}$ Corresponding author.

E-mail david.tsui@utoronto.ca; fax (416) 978-2666.

Article is online at http://www.learnmem.org/cgi/doi/10.1101//m.1188208. more recent study concluded that the age-associated decline in chemotaxis results from a decline in locomotor abilities during aging, and not sensory function deficits (Glenn et al. 2004).

We have analyzed chemotaxic behaviors at ages day 4 and day 7 with different concentrations of odorants. While there was no change in chemotaxis to low concentrations of odorants in the early aging stage, a prolonged attraction to high concentrations of benzaldehyde was observable in day 7 animals compared with day 4 animals. This prolonged attraction also was evident in response to diacetyl, but no change in octanol avoidance was observed. These results suggest that the prolonged attraction to high concentrations of odorants in aged animals is due to a stronger attraction to, rather than a weaker avoidance of, the odorant. This increased attraction was abolished by replacement of $E$. coli with axenic media starting at early adult stage, but reappeared when animals then were returned to $E$. coli food or when benzaldehyde was added to the axenic media. Finally, serotonindeficient mutants were defective in this increased attraction. We therefore conclude that the increased attraction in aged C. elegans is experience dependent, requires serotonin, and may be accounted for by associative learning.

\section{Materials and Methods}

\section{Strains and media}

The wild-type Bristol N2 and CX 2205 odr-3 (n2150), GR1321 tph-1 (mg280), MT9668 mod-1 (ok103), XA406 ncs-1 (qa406), and RB816 sra-11 (ok630) strains were obtained from the Caenorhabditis Genetics Center at the University of Minnesota. UT2 lrn-2 (mm99) animals were isolated and characterized in our lab and frozen at $-80^{\circ} \mathrm{C}$. Worms were well-fed and cultivated at $20^{\circ} \mathrm{C}$ on normal growth medium (NGM) $(50 \mathrm{mM} \mathrm{NaCl}, 15 \mathrm{~g} / \mathrm{L}$ agar, $20 \mathrm{~g} / \mathrm{L}$ peptone, $1 \mathrm{mM}$ cholesterol, $1 \mathrm{mM} \mathrm{CaCl}, 1 \mathrm{mM} \mathrm{MgSO}_{4}, 1 \mathrm{mM}$ $\mathrm{KPi} \mathrm{pH}=7.0$ ) seeded with the OP50 E. coli strain. 


\section{Axenic culture of $C$. elegans}

Adult animals were washed off from a thin food lawn of $E$. coli, allowed to settle and resuspended in distilled water, and repeatedly washed for five times to rid of residual E. coli. Animals were then inoculated to axenic media, and moved to new media every day. Axenic culture media (Chitwood et al. 1995) contained $3 \mathrm{~g}$ of yeast extract, $3 \mathrm{~g}$ of soy peptone, $1 \mathrm{~g}$ of dextrose, $90 \mathrm{~mL}$ of distilled water, and $200 \mu \mathrm{L}$ of a cholesterol stock solution (stock solution is $5 \mathrm{mg} / \mathrm{mL}$ in ethanol), and autoclaved for $30 \mathrm{~min}$. After cooling, hemoglobin stock solution was added to the axenic culture medium in a 1:10 (hemoglobin:media) proportion. Hemoglobin stock solution was prepared by dissolving $5 \mathrm{~g}$ of hemoglobin in $100 \mathrm{~mL}$ of distilled water and filtered. Ampicillin (100 $\mu \mathrm{g} / \mathrm{mL}$ ) was added to prevent any residual bacterial growth.

\section{Chemotaxis assays}

Chemotaxis assays were carried out as previously described (Nuttley et al. 2001). The 10-cm Petri dishes were filled with $6 \mathrm{~mL}$ of agar medium (without E. coli or axenic bacteria). On one side of the agar, a $1-\mu \mathrm{L}$ drop of benzaldehyde (diluted in ethanol) was presented, and $1 \mu \mathrm{L}$ of ethanol was presented on the other side. Prior to this test, $1 \mu \mathrm{L}$ of $1 \mathrm{M} \mathrm{NaN}_{3}$ was applied to the centers of the two test spots to immobilize the animals. The addition to both of the odorants and vehicle sides assured that the chemotaxis to odorants by the animals could not be attributed to the presence of $\mathrm{NaN}_{3}$. After $1 \mathrm{~h}$, a chemotaxis index (C.I.) was calculated to measure their preference toward the test odorant. The C.I. is calculated as the number of animals within 2 $\mathrm{cm}$ of the test spot, minus the number of animals within $2 \mathrm{~cm}$ of the vehicle spot, and then divided by the total number of animals on the plate (Fig. 1A). A positive C.I. indicates an attraction, and a negative C.I. indicates an aversion.

\section{Benzotaxis time-course assay}

For kinetic analysis of chemotaxis and for approaches to $100 \%$ benzaldehyde, a modified grid was used and the $\mathrm{NaN}_{3}$ was omitted so that animals could leave a spot after an initial approach (Nuttley et al. 2001). This grid consisted of four parallel lines drawn 18-mm apart to divide the plate area into five sectors, symmetrical about the third (Fig. 1B). A total of $2 \mu \mathrm{L}$ of benzaldehyde was placed on one of two small pieces of parafilm that had been placed at opposite edges of the agar. At the indicated time points, animals were immobilized by cooling the plates for $2 \mathrm{~min}$ at $-20^{\circ} \mathrm{C}$, and the plates were maintained at $4^{\circ} \mathrm{C}$ until counting. The number of animals in sectors A-E, with the test odorant being in A, were counted, and a weighted C.I. (W.C.I.) was calculated as $([2 \times$ no. in $\mathrm{A}+$ no. in B] $-[2 \times$ no. in $\mathrm{E}+$ no. in $\mathrm{D}]) /($ total number of animals on the plate), yielding a W.C.I. range of from +2.0 to -2.0 . The W.C.I. provides better spatial resolution for the measure of worm behavior in addition to the improved temporal resolution in the benzotaxis assay. Those animals that crawled up the side of the plate were considered lost, and no attempt was made to count these. The score for each plate of 100-300 animals is one data point.

\section{Locomotor assay}

For kinetic analysis of locomotion, a grid with four concentric circles was used (Fig. 1C). Animals were placed at the center of the grid and allowed to disperse freely. At the indicated time points, animals were immobilized by cooling the plates for $2 \mathrm{~min}$ at $-20^{\circ} \mathrm{C}$, and the plates were maintained at $4^{\circ} \mathrm{C}$ until counting. A locomotor index was calculated as $(0 \times$ no. in $\mathrm{A}+1 \times$ no. in $\mathrm{B}+2 \times$ no. in $\mathrm{C}+3 \times$ no. in $\mathrm{D}) /($ total number of animals on the plate), yielding a W.C.I. range of from 0 to 3 . Those animals that crawled up the side of the plate were considered lost, and no attempt was made to count these. The score for each plate of 100-300 animals is one data point.

\section{Dye-filling assay}

Dye-filling using DiI (Molecular Probes) was performed as described previously (http://info.med.yale.edu/mbb/koelle/ protocols/protocol_dye_filling_sens.html).

\section{Statistics}

All C.I. and W.C.I. values are means and standard errors of the means calculated from at least three chemotaxis test plates (sample sizes are stated in figure captions). Two-way ANOVAs
A

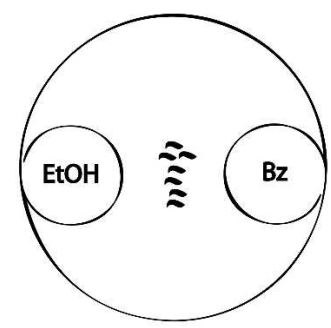

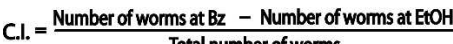
Total number of worms

C

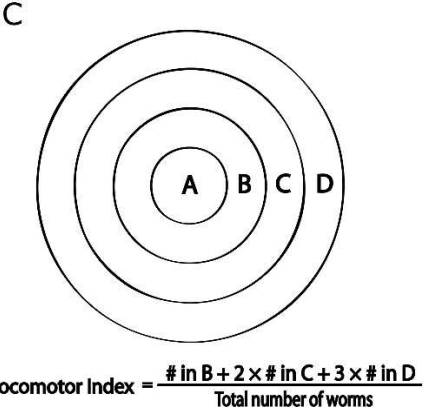

Figure 1. (A) Schematic of the standard chemotaxis assay. Animals' preference for different concentrations of benzaldehyde was tested in the absence of food. Worms were placed at the center of the chemotaxis plate. A 1- $\mu \mathrm{L}$ drop of benzaldehyde of specific concentrations (diluted in ethanol) was presented on one side and a 1- $\mu \mathrm{L}$ drop of ethanol was presented on the other. Animals that approached close to the odorant or ethanol spot were immobilized by $\mathrm{NaN}_{3}$. After $1 \mathrm{~h}$, the chemotaxis index (C.I.) was calculated according to the formula below the diagram. (B) Schematic of the kinetic analysis. Kinetic analysis of chemotaxis to $100 \%$ odorants. Worms were placed at the center of the chemotaxis plate, and a $2-\mu \mathrm{L}$ drop of test odorant was presented in A. Different testing groups were allowed to chemotaxis for different lengths of time, and the number of animals in each sector from $A$ to $\mathrm{E}$ was counted. A weighted C.I. (W.C.I.) was calculated according to the formula below this diagram. (C) Schematic of the locomotion analysis. Locomotion analysis of day 4 vs. day 7 worms. Worms were placed at the center of the agar plate $(A)$ and allowed to roam for different lengths of time. The number of animals in each sector from A to D was counted. A locomotor index was calculated according to the formula below this diagram. 
were performed, followed by Bonferroni-corrected multiple comparisons. The level of significance for all comparisons was $P<0.05$.

\section{Results}

\section{Aged $C$. elegans chemotax more to high concentrations of benzaldehyde than young adults}

In choosing the ages of $C$. elegans for our comparative analysis, two issues were taken into consideration. First, we had to minimize the possibility of phenotypic differences in development that could account for any behavioral difference between the two ages compared. When grown at $20^{\circ} \mathrm{C}$, C. elegans proceed through four larval stages. They lay their first egg $\sim 3 \mathrm{~d}$ after hatching, and they will lay the majority of their eggs in the following 3-4 d (Evason et al. 2005). Second, we needed to minimize the possibility of locomotor deficits in aged animals. At day 7, animals start to show loss of muscle integrity, including misshapen nuclei, appearances of small patches in nuclei, and patched and wrinkled sarcomeres (Herndon et al. 2002; Glenn et al. 2004). Spontaneous movements and pharyngeal pumping also begin to decline at this age (Glenn et al. 2004; Chow et al. 2006). However, using a locomotor assay on a modified grid (Fig. 1C), we found no significant difference in the rate of locomotion between day 4 and day 7 animals (Fig. 2B). There was no significant age $\times$ time interaction $\left(F_{(2,12)}=0.4, P>0.05\right)$ nor a significant main effect of age $\left(F_{(1,12)}=0.002, P>0.05\right)$, but a significant main effect of time $\left(F_{(2,12)}=96.15, P<0.05\right)$ was seen. Therefore, the analysis was done between day 4 and day 7 animals, a period that marks approximately the beginning and end of the reproductive period.

To examine whether day 4 and day 7 animals have different chemotaxic behavior, animals were tested on standard chemotaxis assays (Bargmann et al. 1993) using different concentrations of the odorant benzaldehyde. Briefly, benzaldehyde of specific dilution was applied to one side of the chemotaxis plate, and the ethanol vehicle was applied to the other side (Fig. 1A). All chemotaxis assays performed in this study were performed on plain agar plates in the absence of $E$. coli or worm growth medium. Animals were put in the middle of the testing plate and allowed to roam for $1 \mathrm{~h}$. Prior to the addition of odorant and vehicle, $\mathrm{NaN}_{3}$ was applied to both sides of the plate to immobilize the animals that approach close enough to the odorant or vehicle spot. A two-factor analysis of variance (ANOVA) revealed a significant age $\times$ concentration interaction $\left(F_{(5,24)}=20.09, P<0.05\right)$. When tested in this assay with low benzaldehyde concentrations $(0.01 \%, 0.05 \%, 0.1 \%$, and $1 \%)$, no difference was observed between day 4 and day 7 animals (Fig. $2 \mathrm{~A})$. This is consistent with a previous study that showed no difference in chemotaxis to $1 \%$ benzaldehyde between young and aged adults (Glenn et al. 2004). However, when tested with higher concentrations (10\% and $100 \%)$ of benzaldehyde in the present study, day 7 animals showed a significantly higher chemotaxis index (C.I.) compared with day 4 animals. This result suggests that there is a change in the chemotaxic behavior as a result of a change in the initial sensitivity or in other downstream components of the chemotaxis pathway, specifically to high concentrations of benzaldehyde.
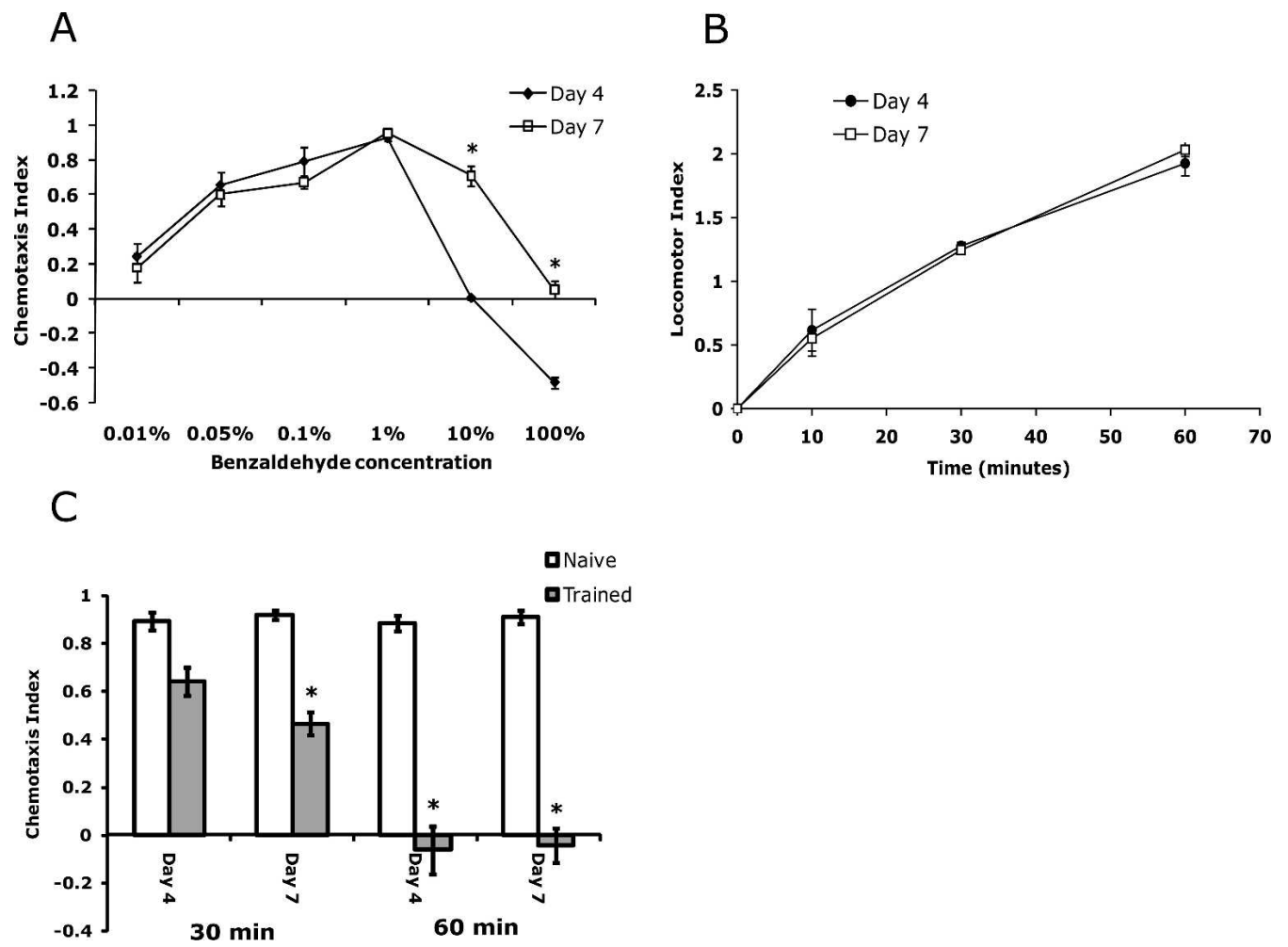

Figure 2. (A) Chemotaxis by Caenorhabditis elegans. Chemotaxis index (C.I.) after $1 \mathrm{~h}$ of chemotax to $0.01 \%, 0.05 \%, 0.1 \%, 1 \%, 10 \%$, and $100 \%$ benzaldehyde ( $n=3$ plates per data point) for day $4(\bullet)$ and day 7 animals $(\square)$. A positive C.I. indicates an attraction to the odor; a negative score indicates an aversion. $\left.{ }^{*}\right)$ Significant difference in C.I. between day 4 and day 7 animals $(P<0.05$, Bonferroni-corrected $t$-test). (B) Locomotion of day 4 vs. day 7 animals. Time course for locomotion by day $4(\bullet)$ and day $7(\square)$ wild-type animals. $n=6$ plates per data point for all data points. ( $\left.{ }^{*}\right)$ Significant difference in W.C.I. between groups at the same time point $(P<0.05$, Bonferroni-corrected $t$-test). (C) Olfactory adaptation of day 4 vs. day 7 animals. Chemotaxis index to $1 \%$ benzaldehyde for animals that received no exposure (naive; hollow bars) or were exposed to $100 \%$ benzaldehyde (trained; gray-filled bars) for the duration (30 or $60 \mathrm{~min}$ ) stated below each set of bars. The ages of the groups also are stated below each set of bars. $n=9$ plates per data point. $\left(^{*}\right)$ Significant difference in C.I. between naive and trained group $(P<0.05$, Bonferroni-corrected $t$-test). 
Higher chemotaxis to high concentration of benzaldehyde in aged adults is due to a prolonged attraction to benzaldehyde and not a reduction in the ability to discriminate between odorant concentrations

The lack of a negative chemotaxis at high concentrations in day 7 adults suggests the possibility that there is a reduction in the ability to discriminate between odorant concentrations in aged animals. To test this hypothesis, we performed the olfactory adaptation assay (Coburn and Bargmann 1996) on day 4 and day 7 animals. In this assay, animals were trained to $100 \%$ benzaldehyde in the absence of food and tested to $1 \%$ benzaldehyde. Day 7 animals showed a similar naive approach to day 4 animals (Fig. 2C). A three-way ANOVA (age $\times$ treatment $\times$ duration) revealed a significant treatment (naive vs. trained) $\times$ duration $(30 \mathrm{~min}$ vs. $60 \mathrm{~min})$ interaction $\left.\left(F_{(1,6}\right)=58.12, P<0.05\right)$. Post-hoc Bonferroni $t$-tests indicated that day 7 animals showed significantly lower chemotaxis to $1 \%$ benzaldehyde after $30 \mathrm{~min}$ of training to $100 \%$ benzaldehyde off food (i.e., adaptation; $P<0.05$ ), while day 4 animals showed an insignificant trend of adaptation $(P>0.05)$. However, after 60 min of training, both day 4 and day 7 animals showed a significant reduction in chemotaxis $(P<0.05)$ of similar magnitude. These results suggest that day 7 animals may adapt to benzaldehyde at a slightly faster rate compared with day 4 animals, but the higher chemotaxis to high concentrations in day 7 animals is not likely due to a reduction in the ability of the aged worms to discriminate between odorant concentrations.

A previous study demonstrated that $C$. elegans respond to $100 \%$ benzaldehyde in a biphasic manner (Nuttley et al. 2001). Animals initially approach the spot of benzaldehyde for about 15 min, resulting in an increasing C.I. with time. After $15 \mathrm{~min}$, they begin to show a strong avoidance to benzaldehyde, resulting in a decreasing and then a negative C.I. To ask which part of this curve is responsible for the higher C.I. observed in aged animals, a kinetic analysis of chemotaxis behavior was performed on day 4 and day 7 animals. The paradigm of the kinetic analysis is similar to the standard chemotaxis paradigm, except that $\mathrm{NaN}_{3}$ was not applied in this assay. Instead, worms were allowed to roam freely and plates were frozen at $-20^{\circ} \mathrm{C}$ to immobilize the worms at specific time points during the assay. A stronger and more prolonged attraction (hence, a delayed avoidance) was observed in day 7 animals compared with day 4 animals (Fig. 3A). There was an age $\times$ time interaction $\left(F_{(5,24)}=4.57, P<0.05\right)$. Day 7 animals showed significantly higher weighted chemotaxis index (W.C.I.) compared with day 4 animals at all time points. This result suggests that day 7 animals showed a much stronger attraction to benzaldehyde compared with day 4 animals. Behaviorally, on a population level, day 7 animals approached benzaldehyde quicker and clustered around the spot of benzaldehyde for a longer time period. Day 4 animals also showed an initial attraction to the spot of benzaldehyde, but they were dispersed over a larger area and did not approach the benzaldehyde spot as closely as the day 7 animals did.

\section{Prolonged attraction in aged adults is due to a stronger attraction and not a weaker avoidance}

There are distinct mechanisms that mediate the attractive and the aversive benzaldehyde chemotaxis responses (Nuttley et al. 2001); therefore, two alternative explanations exist for the prolonged benzaldehyde attraction. One hypothesis is that day 7 animals had a stronger attraction, and therefore they approached quicker and stayed closer to the spot of benzaldehyde. An alternative hypothesis is that day 7 animals had a weaker avoidance, and therefore were not repelled by the benzaldehyde as much as the day 4 animals were, thus avoiding benzaldehyde much slower than day 4 animals. To determine which hypothesis is more plausible, a mutant strain defective in the initial attractive behavior to benzaldehyde (Roayaie et al. 1998; Nuttley et al. 2001) was tested for any age-related difference in chemotaxis behavior. odr-3 encodes a G-protein $\alpha$ subunit that is expressed in AWA, AWB, AWC, ASH, and ADF neurons and is required for behavioral responses known to be mediated by these sensory neurons (Troemel et al. 1995; Roayaie et al. 1998; Fukuto et al. 2004). The $o d r-3(n 2150)$ mutant is defective in attraction to benzaldehyde, while avoidance to benzaldehyde was comparable to $\mathrm{N} 2$ both in time and in magnitude (Nuttley et al. 2001). Thus, it was concluded that the odr-3 gene is required for the approach to benzaldehyde, but is not necessary for the avoidance response. Therefore, a kinetic analysis of benzaldehyde chemotaxis was performed for day 4 and day 7 odr-3 animals.

Interestingly, the prolonged attraction to benzaldehyde was abolished in day 7 odr-3 animals (Fig. 3B). There was no age $\times$ time interaction $\left(F_{(5,24)}=2.57, P>0.05\right)$, but significant main effects of age $\left(F_{(1,24)}=6.21, P<0.05\right)$ and time $\left(F_{(5,24)}=62.82\right.$, $P<0.05)$ were seen. To our surprise, the time-course chemotaxis curves had reversed their pattern, such that the day 7 animals showed a more negative W.C.I. at 30 min when compared with day 4 animals. Thus, when the attractive phase of benzaldehyde
A

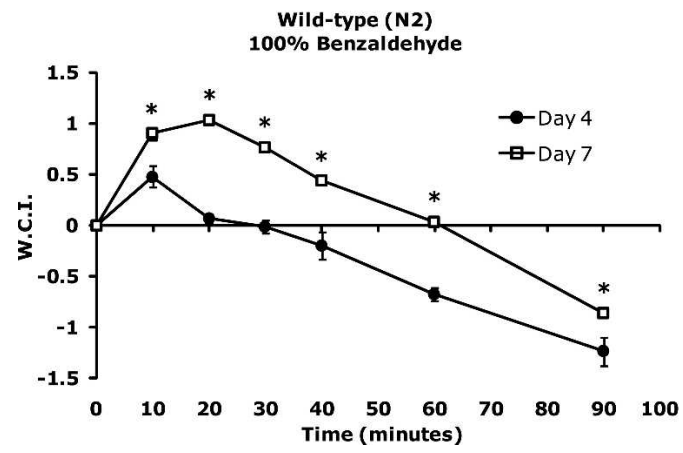

B

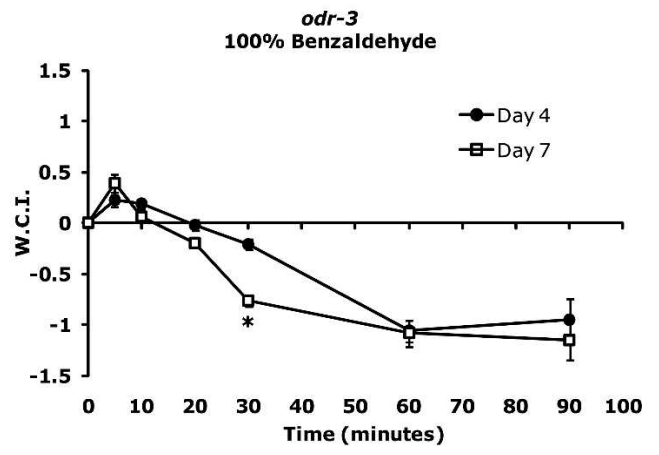

Figure 3. Time course for chemotaxis to $100 \%$ benzaldehyde. (A) Time course for chemotaxis behavior of wild-type N2 animals. (B) Time course for chemotaxis behavior of odr-3(n2150), a mutant strain that is defective in the attractive response to benzaldehyde. Weighted chemotaxis index (W.C.I.) is shown after various time points of chemotaxis to $100 \%$ benzaldehyde $(n=3$ plates per data point) for day $4(\bullet)$ and day 7 ( $\square)$ animals. A positive W.C.I. indicates an attraction to the odor; a negative score indicates an aversion. ( $\left.{ }^{*}\right)$ Significant difference in W.C.I. between day 4 and day 7 animals $(P<0.05$, Bonferroni-corrected $t$-test). 
chemotaxis was abolished, not only was prolonged attraction abolished in day 7 animals, but day 7 animals also avoided the spot of benzaldehyde quicker. However, this quicker avoidance may be secondary to day 7 animals exposed to a higher dose of benzaldehyde by virtue of approaching closer to the odorant spot. This result suggests that the prolonged attraction observed in aged wild-type animals was caused by a stronger attraction rather than a weaker avoidance.

\section{Young and aged adults approach low concentrations} of benzaldehyde with similar kinetics and magnitude We reasoned that any general change in benzaldehyde sensitivity should be most apparent when testing with low concentrations of odorant. Although no age-dependent difference in C.I. was observed for chemotaxis to low concentrations of benzaldehyde when tested with the standard chemotaxis assay (Fig. 2A), the C.I. was cumulative over the 1-h period due to the application of $\mathrm{NaN}_{3}$ to capture the animals, and thus may not be sensitive enough to reveal any minute change in chemotaxic response. We therefore asked whether there is any difference in kinetics of approach to low concentrations of benzaldehyde. No difference was observed in the rate of approach to $0.1 \%$ benzaldehyde between day 4 and day 7 animals (Fig. 4A). There was no significant age $\times$ time interaction $\left(F_{(3,16)}=2.81, P>0.05\right)$, nor a significant main effect of age $\left(F_{(1,16)}=0.002, P>0.05\right)$, but a significant main effect of time $\left(F_{(3,16)}=77.74, P<0.05\right)$ was seen. However, there was a quicker approach to $1 \%$ benzaldehyde in day 7 animals (Fig. 4B). There was a significant age $\times$ time interaction $\left(F_{(3,16)}=8.84\right.$, $P<0.05)$, and the W.C.I. for day 7 animals was significantly higher than that of the day 4 animals at the 5-min time point.

The approach to $1 \%$ benzaldehyde was analyzed in odr-3 mutant animals as well. Consistent with a previous report (Roayaie et al. 1998), the approach to $1 \%$ benzaldehyde was significantly reduced in odr-3 mutants (Fig. 4C). In addition, the

A
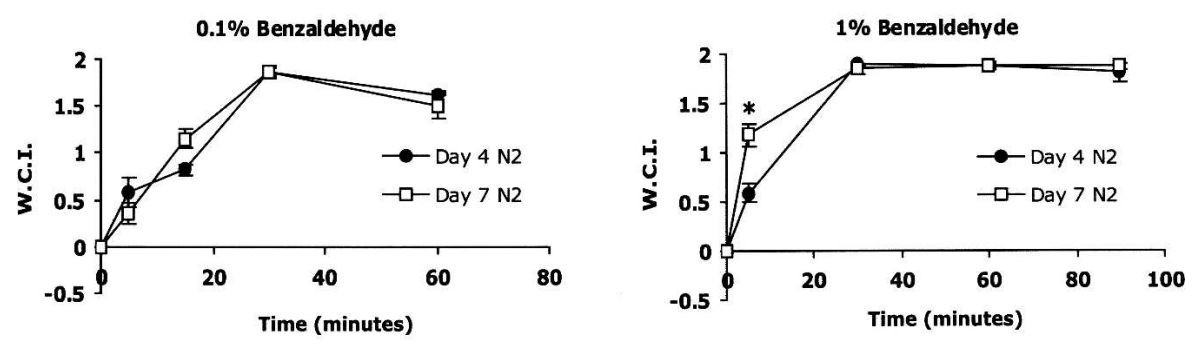

C

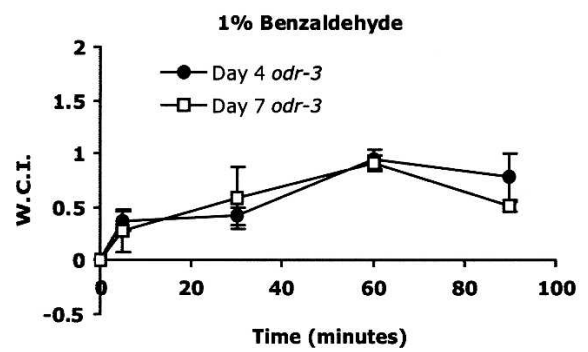

Figure 4. Chemotaxis behavior to low concentrations of benzaldehyde. (A) Time course for approach to $0.1 \%$ benzaldehyde by day 4 and day 7 wild-type animals $(n=3$ plates per data point). $(B, C)$ Time course for approach to $1 \%$ benzaldehyde by day 4 and day 7 (B) wild-type and (C) odr-3 (n2150) animals ( $n=3$ plates per data point). ( $\bullet$ Day $4 ;(\square)$ day 7. ( $\left.{ }^{*}\right)$ Significant difference in W.C.I. between day 4 and day 7 animals $(P<0.05$, Bonferroni-corrected $t$-test). quicker approach to $1 \%$ benzaldehyde by wild-type day 7 animals compared with wild-type day 4 animals was abolished in odr-3 animals. There was no significant age $\times$ time interaction $\left(F_{(3,16)}=0.62, P>0.05\right)$ nor significant main effect of age $\left(F_{(1,16)}=0.22, P>0.05\right)$, but a significant main effect of time $\left(F_{(3,16)}=5.07, P<0.05\right)$ was seen. Therefore, when the attraction was deficient with the mutation of $o d r-3$, the enhanced attraction to $1 \%$ benzaldehyde was no longer seen, reinforcing the hypothesis that the prolonged attraction to benzaldehyde was due to a stronger attractive mechanism. Together, these data suggest that there is a stronger attraction in aged animals that is specific to high concentrations of benzaldehyde ( $1 \%$ or higher).

\section{Stronger benzaldehyde chemotaxis in aged adults generalizes to attractive odorants but not aversive odorants}

To ask whether the stronger expression of the chemotaxis response to $100 \%$ benzaldehyde in day 7 animals compared with day 4 animals exists for other volatile odorants, kinetic analyses of chemotaxis to diacetyl and octanol were performed. Diacetyl is another attractive odorant for $C$. elegans and is sensed by the AWA primary chemosensory neurons (Bargmann et al. 1993; Sengupta et al. 1996). Octanol is an aversive odorant and is sensed by the AWB primary chemosensory neurons, which mediate olfactory avoidance behavior in C. elegans. The response to $100 \%$ octanol in the absence of food was found to be mediated by the AWB primary sensory neurons as well as the ASH and ADL mechanosensory neurons (Troemel et al. 1995; Chao et al. 2004).

The paradigm used for diacetyl chemotaxis was identical to that for benzaldehyde. A prolonged attraction to 100\% diacetyl in day 7 animals compared with day 4 animals was observed, similar to that seen with $100 \%$ benzaldehyde (Fig. 5A). There was a significant age $\times$ time interaction $\left(F_{(4,70)}=3.09, P<0.05\right)$. Day 7 animals showed a significantly higher W.C.I. at 10, 30, 60, and 90 min compared with day 4 animals. This result suggests that similar to the response to benzaldehyde, aged animals also showed a stronger attraction to $100 \%$ diacetyl compared with young adults.

The kinetic analysis of chemotaxis to octanol was performed with the same paradigm, except that the worms were positioned immediately beside the spot of octanol at the beginning of the assay because octanol only elicits an aversive response (Troemel et al. 1995). Putting the worms beside the spot of odorant near one edge of the testing plate allowed for a longer distance (the entire diameter of the plate) for avoidance of the spot of octanol, rather than half the diameter of the plate when worms started their chemotaxis at the center of the plate. This should provide a better resolution for the kinetics of octanol avoidance. Interestingly, no difference in rate of avoidance was observed between day 4 and day 7 animals (Fig. 5B). There were no significant age $\times$ time interaction $\left(F_{(5,24)}=0.53, P>0.05\right)$ nor significant main effect of age $\left(F_{(1,24)}=3.08\right.$, $P>0.05)$, but there was a significant main effect of time $\left(F_{(5,24)}=20.41\right.$, $P<0.05)$. Taken together, these data 
A

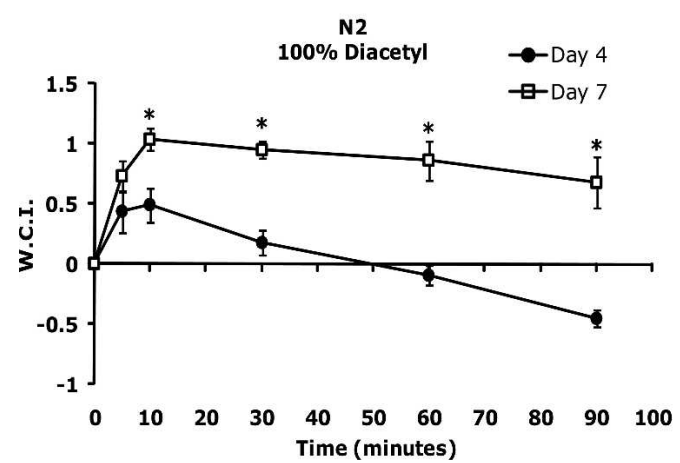

B

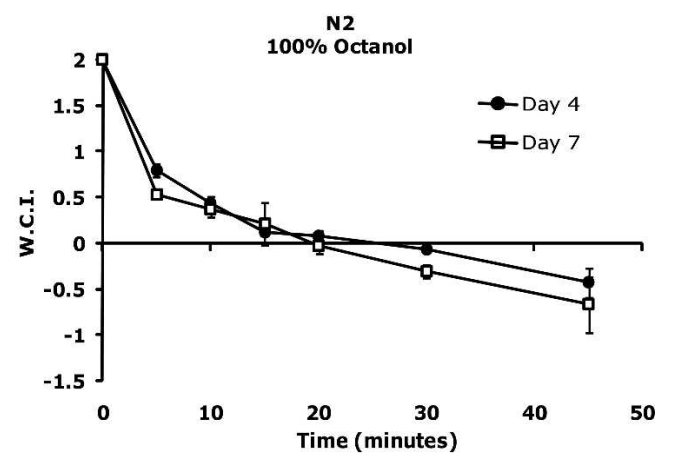

Figure 5. Chemotaxis behavior to other odorants. Time course for chemotaxis to $(A) 100 \%$ diacetyl ( $n=8$ plates per data point), and $(B) 100 \%$ octanol ( $n=3$ plates per data point) by day $4(\bullet)$ and day $7(\square)$ wild-type animals. Animals were placed in the center of the plate for chemotaxis to diacetyl and immediately beside the odorant spot for octanol at the start of the chemotaxis assay. ${ }^{*}$ ) Significant difference in W.C.I. between day 4 and day 7 animals $(P<0.05$, Bonferroni-corrected $t$-test).

suggest that the stronger chemotaxis in aged worms generalizes to odorants that have an attractive component, but not to odorants that have only an aversive component.

\section{Stronger benzaldehyde attraction by day 7 animals is experience dependent}

To test whether the age-enhanced attraction to benzaldehyde correlates with the age of animals, 4-, 6-, and 8-d-old animals were tested for their chemotaxis response. Indeed, stronger chemotaxis response was observed in progressively older animals (Fig. 6A). There was an age $\times$ time interaction $\left(F_{(6,60)}=2.65\right.$, $P<0.05)$. Day 6 animals showed higher C.I. compared with day 4 animals at all time points $(P<0.05)$, and day 8 animals showed higher C.I. compared with day 6 animals at the 10-, 30-, and 60 -min time points $(P<0.05)$. This suggests that the ageenhanced attraction is indeed correlated with the animal's age, and that the strength of the attraction may be dependent on length of food exposure.

Benzaldehyde and diacetyl are both biologically relevant olfactory cues for C. elegans. Benzaldehyde is thought to be produced by bacteria (http://www.sciencedaily.com/releases/2001/ 04/010405081652.htm) and has been found to be produced by some bacteria through conversion from phenylalanine (Nierop Groot and de Bont 1998). Diacetyl is also a bacterial fermentation end product from a food source for C. elegans (Zhang et al. 1997). Therefore, these volatile odorants may provide a cue for longrange detection of bacterial lawns, and have been shown to play a role in associative and nonassociative learning (Bernhard and van der Kooy 2000; Nuttley et al. 2002).

We hypothesize that by virtue of living on a bacterial lawn for three extra days compared with day 4 animals, day 7 animals had a longer acquisition period to associate the volatile odorants released by bacteria with the presence of the bacterial food source itself, and therefore demonstrated increased expression of attraction. This hypothesis was tested by growing worms in axenic growth media (Chitwood et al. 1995) instead of on E. coli. Axenic growth media support worm growth in the absence of bacterial food source; hence, the metabolites that are released by bacterial food are absent in this growth condition, while worms still grow into adults on this food. In this growth condition, the contingency between olfactory stimuli (bacterial metabolites including benzaldehyde and diacetyl) and the presence of food should be removed.

To test this hypothesis, we replaced the E. coli food with axenic media after worms were grown to adults on $E$. coli, and then tested their chemotaxic response to benzaldehyde. Worms grown on axenic media for $3 \mathrm{~d}$ after $4 \mathrm{~d}$ of growth on E. coli (referred to as the axenic media group below) showed a weaker attraction to benzaldehyde compared with animals grown for $7 \mathrm{~d}$ on $E$. coli (Fig. 6B). A two-way ANOVA reveals a significant treatment $\times$ time interaction $\left(F_{(6,24)}=4.07, P<0.05\right)$ in the chemotaxis time course. Post-hoc Bonferroni $t$-tests indicated that the day 7 E. coli alone group showed significantly more attraction to benzaldehyde than the day $4 E$. coli alone group and the axenic media group $(P<0.05)$. Interestingly, animals that were returned to $E$. coli food source for $2 \mathrm{~d}$ after $4 \mathrm{~d}$ of $E$. coli growth and $1 \mathrm{~d}$ of axenic media growth showed a chemotaxic behavior that was not significantly different from animals grown on E. coli for $7 \mathrm{~d}$ alone $(P>0.05)$ and C.I. that was significantly higher than day 4 E. coli alone animals at 30 and $60 \mathrm{~min}(P>0.05)$. Most important, a locomotor assay revealed no difference in the locomotion of all treatment groups (Fig. 6D) $\left(F_{(4,14)}=0.29, P>0.05 ; P>0.05\right.$ for all comparisons between any two treatment groups by Bonferroni's multiple comparison test), suggesting that the differences in attraction between different groups cannot be attributed to locomotor differences. These data suggest that the odor-food association was extinguished by the loss of the odor-food contingency in animals that were grown on axenic media, and is reversible upon re-exposure to the $E$. coli food source. However, animals that were returned to the $E$. coli food source for $1 \mathrm{~d}$ after $4 \mathrm{~d}$ of $E$. coli growth and $2 \mathrm{~d}$ of axenic media growth showed a nonstatistically significant trend of greater attraction to benzaldehyde (data not shown), suggesting that the enhanced attraction correlates with the length of odor-food exposure. Thus, these results support the idea that the increased benzaldehyde attraction in day 7 animals is dependent on experience, specifically the contingency between odor metabolites released from $E$. coli and the appetitive value of food itself. Furthermore, these results suggest that the attraction to high concentrations of odorants remains plastic during adult stage and may be modified based on the associatively learned contingencies between sensory stimuli and appetitive cues present in the environment.

\section{Reconstitution of the benzaldehyde-food contingency in axenic media restores age-enhanced attraction to benzaldehyde}

If the enhanced attraction is indeed due to the association between odor given off food and food itself, one should predict that supplementing axenic media with benzaldehyde should restore the benzaldehyde-food contingency and hence the ageenhanced attraction to benzaldehyde. To further test the hypothesis that the aged-enhanced attraction is due to odor-food association, animals were given a supplement of $0.06 \%$ benzaldehyde 
A

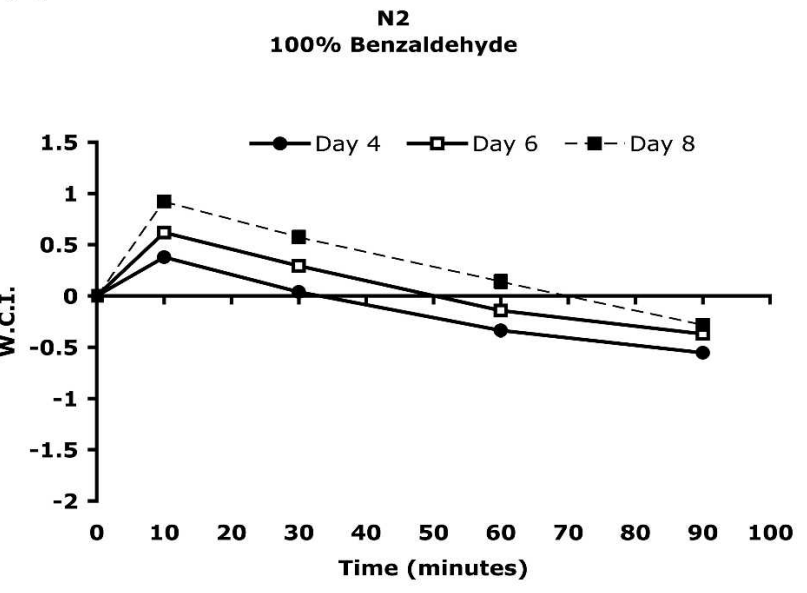

C

N2

$100 \%$ Benzaldehyde

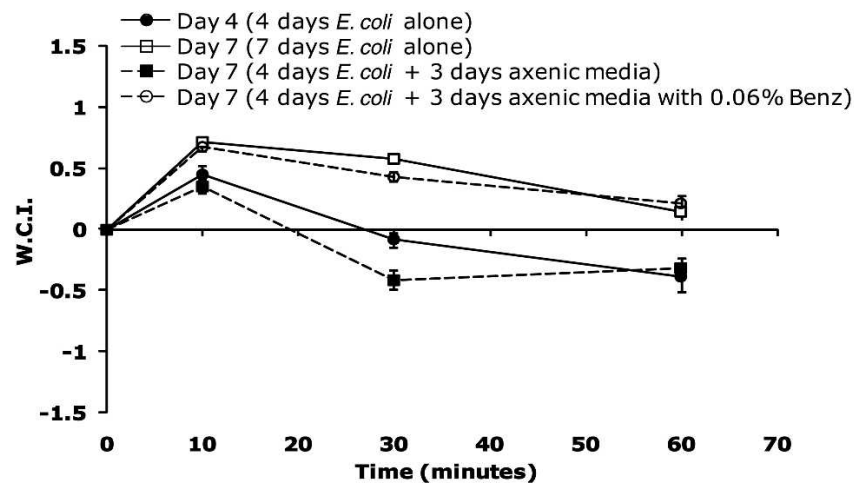

B

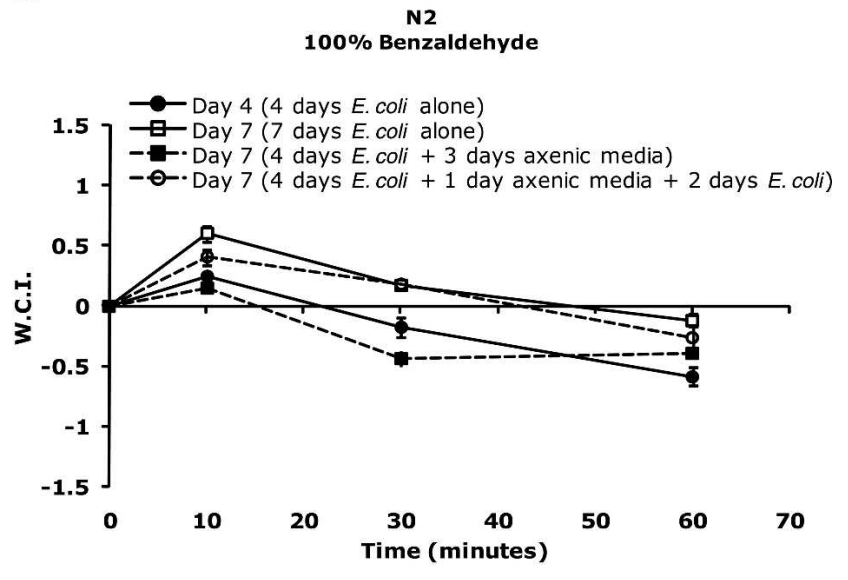

$\mathrm{D}$

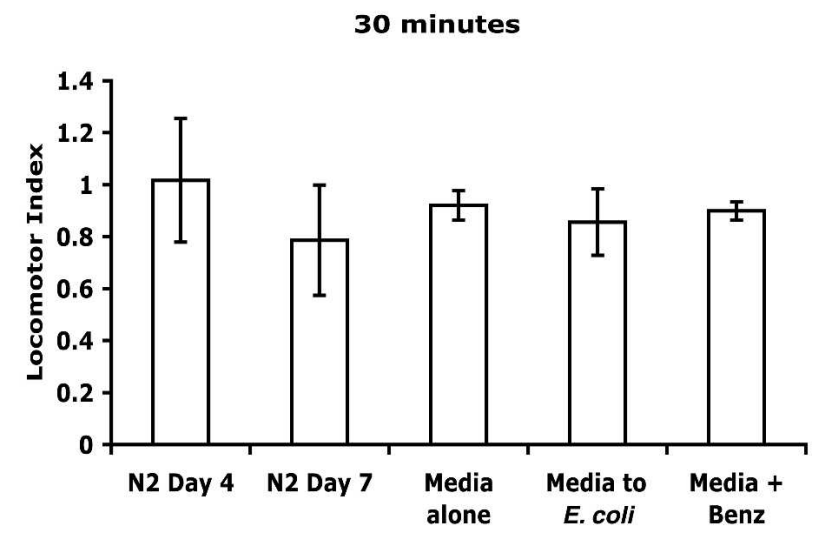

Figure 6. (A) Chemotaxis behavior of progressively older animals. Time course for chemotaxis to $100 \%$ benzaldehyde by day $4(\bullet)$, day $6(\square)$, and day 8 ( $\square$ with dashed line) wild-type animals. $n=6$ plates per data point for all data points. $(B, C)$ Chemotaxis behavior of worms grown on axenic media. (B) Time course for chemotaxis to $100 \%$ benzaldehyde by wild-type animals grown for (1) $4 \mathrm{~d}$ on $E$. coli (๑), (2) $7 \mathrm{~d}$ on $E$. coli ( $\square)$, (3) $4 \mathrm{~d}$ on $E$. coli +3 $\mathrm{d}$ on axenic growth media ( $\square)$, and (4) $4 \mathrm{~d}$ on $E$. coli $+1 \mathrm{~d}$ on axenic media $+2 \mathrm{~d}$ on $E$. coli $(O) . n=3$ plates per data point for all data points. (C) Time course for chemotaxis to $100 \%$ benzaldehyde by wild-type animals grown for (1) $4 \mathrm{~d}$ on $E$. coli ( $(2)$, (2) $7 \mathrm{~d}$ on $E$. coli ( $\square$ ), (3) $4 \mathrm{~d}$ on $E$. coli $+3 \mathrm{~d}$ on axenic growth media ( $(\mathbf{D})$, and (4) $4 \mathrm{~d}$ on $E$. coli $+3 \mathrm{~d}$ on axenic media with $0.06 \%$ benzaldehyde $(O) . n=6$ plates per data point for all data points. (D) Locomotion of animals in different treatment groups. Locomotor index at 30 min for wild-type animals that were grown (1) $4 \mathrm{~d}$ on $E$. coli, (2) $7 \mathrm{~d}$ on E. coli, (3) $4 \mathrm{~d}$ on E. coli $+3 \mathrm{~d}$ on axenic growth media, (4) $4 \mathrm{~d}$ on E. coli $+1 \mathrm{~d}$ on axenic media $+2 \mathrm{~d}$ on E. coli, and (4) $4 \mathrm{~d}$ on E. coli $+3 \mathrm{~d}$ on axenic media with $0.06 \%$ benzaldehyde. $n=3$ plates per treatment group.

in the media from day 4 to day 7 after grown to adults on E. coli, and were tested for their chemotaxic behavior to benzaldehyde. Interestingly, this treatment indeed recovered the age-enhanced attraction to benzaldehyde (Fig. 6C). There was a significant age $\times$ time interaction $\left(F_{(6,60)}=6.11, P<0.05\right)$. Animals that received media treatment with a benzaldehyde supplement between day 4 and day 7 showed significantly higher W.C.I. $(P<0.05)$ at all time points compared with both day 4 animals and animals treated with axenic media alone between day 4 and day 7. Moreover, they showed W.C.I. that was not significantly different from day $7 \mathrm{E}$. coli raised animals at all time points $(P>0.05)$. This result further supports the hypothesis that the age-enhanced attraction to benzaldehyde is due to the odor-food association during the animals' exposure to E. coli.

\section{Serotonin is necessary for the enhanced attraction} in aged worms

Serotonin mediates many food-modulated behaviors in C. elegans, including pharyngeal pumping, egg laying, fat metabo- lism, and locomotion (Sawin et al. 2000; Sze et al. 2000), suggesting that serotonin functions as an important signaling molecule for food. Serotonin also has been shown to mediate odor-food associative learning in C. elegans (Nuttley et al. 2002; Zhang et al. 2005). We therefore tested serotonin-deficient mutants to further examine whether the age-enhanced attraction to benzaldehyde could be due to odor-food association. tph-1 encodes tryptophan hydroxylase, which is the rate-limiting enzyme for serotonin synthesis. tph-1 mutants lack the signal for food and therefore should show a deficit in the age-enhanced attraction to benzaldehyde if the increased attraction is caused by the association between odor and food. Indeed, day 7 tph-1 animals lacked the enhanced attraction in day $7 \mathrm{~N} 2$ animals (Fig. 7A) and showed a chemotaxic response that is almost identical to day 4 tph-1 animals (Fig. 7B). There was no significant age $\times$ time interaction $\left(F_{(2,30)}=0.37, P>0.05\right)$ nor a significant main effect of age $\left(F_{(1,30)}=0.66, P>0.05\right)$, but there was a significant maineffect of time $\left(F_{(2,30)}=95.97, P<0.05\right)$ in the $t p h-1$ worms. However, it is possible that an enhanced attraction in aged tph-1animals is 
A

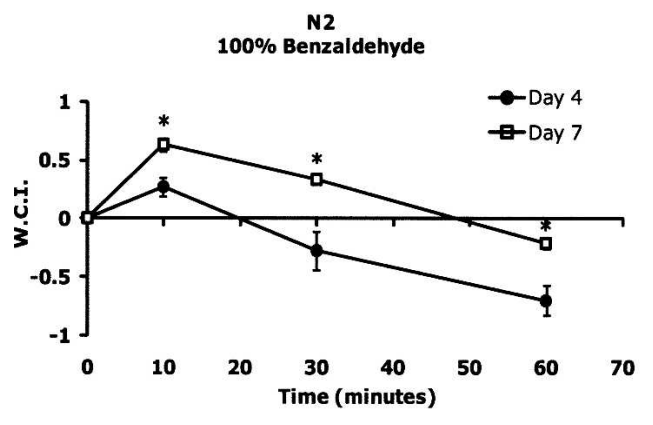

C

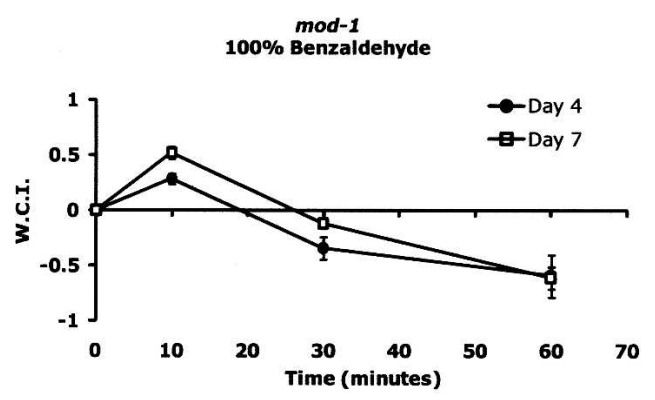

B

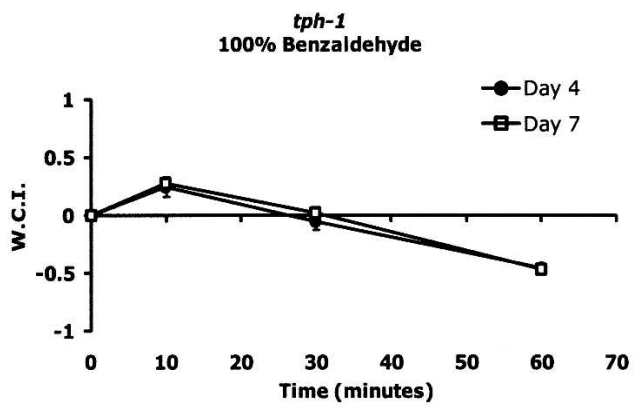

D

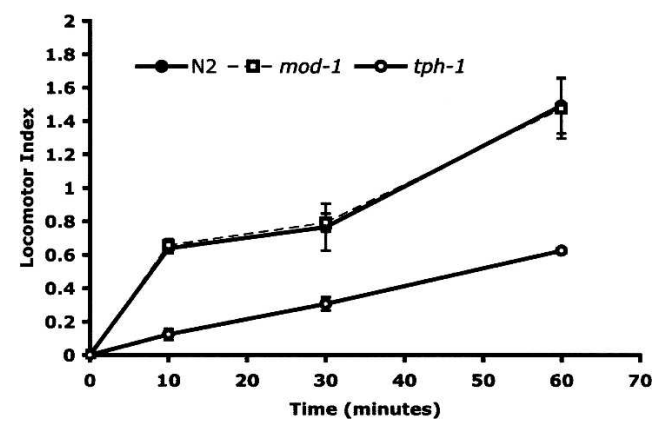

Figure 7. ( $A-C)$ Chemotaxis behavior of serotonin mutants. Time course for chemotaxis to $100 \%$ benzaldehyde by day 4 vs. day 7 animals for three strains: $(A) \mathrm{N} 2$ wild-type animals, $(B)$ tph-1 mutants, and $(C)$ mod-1 mutants. $n=6$ plates per data point for all data points. $\left({ }^{*}\right)$ Significant difference in W.C.I. between groups at the same time point $(P<0.05$, Bonferroni-corrected $t$-test). $(D)$ Locomotion of serotonin mutants. Time course for locomotion by wild-type animals $(\bullet)$, tph-1 mutants $(\bigcirc)$, and mod-1 mutants ( $\square$ with dashed line). $n=3$ plates per data point for all data points.

masked by their deficit in locomotion (Fig. 7D). Although there was no significant strain $\times$ time interaction $\left(F_{(4,18)}=1.52\right.$, $P>0.05)$, a significant main effect of strain $\left(F_{(2,18)}=39.25\right.$, $P>0.05)$ and a significant main effect of time $\left(F_{(2,18)}=44.96\right.$, $P<0.05)$ were seen; tph-1 animals showed a lower locomotor index compared to $\mathrm{N} 2$ at all time points $(P<0.05)$.

mod-1 encodes a serotonin-gated chloride channel and mod-1 mutants are defective in food-modulated locomotor behavior (Ranganathan et al. 2000) and olfactory aversive learning induced by pathogenic food (Zhang et al. 2005). However, unlike tph-1 mutants, mod-1 mutants did not exhibit locomotor deficit in the absence of food (Fig. 7D). The locomotor index of mod-1 animals was not significantly different from N2 at all time points $(P>0.05)$. Therefore, $\bmod -1$ is a good candidate mutant to test for the effect of loss of food signaling on the enhanced attraction to benzaldehyde. Similar to tph-1 mutants, aged mod-1 animals did not show enhanced attraction to benzaldehyde (Fig. 7C). There was no significant age $\times$ time interaction $\left(F_{(2,30)}=0.95\right.$, $P>0.05)$ nor a significant main effect of age $\left(F_{(1,30)}=3.0\right.$, $P>0.05)$, but there was a significant main effect of time $\left(F_{(2,30)}=46.51, P<0.05\right)$, and the W.C.I for day 7 mod- 1 animals was not significantly different from day 4 mod-1 animals at all time points $(P>0.05)$.

Taken together, these results suggest that serotonin is necessary for the age-enhanced attraction to benzaldehyde and may act as a mediator for the food signal in the association between food-released odor and food itself.

\section{Irn-2 is required for the enhanced attraction in aged} worms

To further elucidate the mechanisms of this form of olfactory learning, we tested mutants with deficits in various forms of associative learning in our age-enhancement paradigm. Because
AIY is a downstream target for the olfactory signal from AWC and the food signal from ADF, it is plausible that these sensory signals converge on AIY for the form of associative learning we observed. We therefore tested the involvement of genes that are expressed mainly in AIY neurons and are known to mediate food appetitive learning. The neuronal-Ca ${ }^{2+}$-sensor $n c s-1$ is required for optimal isothermal tracking after worms were raised at the "preferred" temperature in the presence of food (Gomez et al. 2001). sra-11 encodes an orphan $G$ protein-coupled seven-transmembrane receptor that belongs to a large family of putative chemoreceptorencoding genes, and is required for olfactory imprinting, during which larvae $C$. elegans were trained to low concentrations of odorants in the presence of food (Remy and Hobert 2005). The enhanced attraction in aged animals was preserved in ncs-1 mutants (Fig. 8A). There was a significant age $\times$ time interaction in ncs-1 worms $\left(F_{(3,16)}=57.03, P<0.05\right)$ and $\mathrm{N} 2$ control worms $\left(F_{(3,16)}=3.59, P<0.05\right)$. Post-hoc Bonferroni $t$-tests showed that day 7 animals had a higher chemotaxis index than day 4 animals at all time points for both $n c s-1$ and N2 animals $(P<0.05)$. The enhanced attraction in aged animals also was preserved in $s r a-11$ mutants (Fig. 8B). There was a significant age $\times$ time interaction in sra-11 worms $\left(F_{(2,12)}=4.21, P<0.05\right)$ and N2 control worms $\left(F_{(2,12)}=4.28, P<0.05\right)$. Post-hoc Bonferroni $t$-tests showed that day 7 animals had a higher chemotaxis index than day 4 animals at all time points for both sra-11 and N2 animals $(P<0.05)$. However, a deficit is observed in lrn-2 mutants (Fig. 8C), which have been behaviorally characterized and found to be normal in their sensorimotor abilities and nonassociative learning ability, while defective in both olfactory and taste associative learning (Wen et al. 1997; Morrison et al. 1999). There was no significant age $\times$ time interaction $\left(F_{(2,12)}=1.30, P>0.05\right)$ nor a significant main effect of age $\left(F_{(1,12)}=4.61, P>0.05\right)$, but there was a significant main effect of time $\left(F_{(2,12)}=91.97, P<0.05\right)$ in the $l r n-2$ 
A
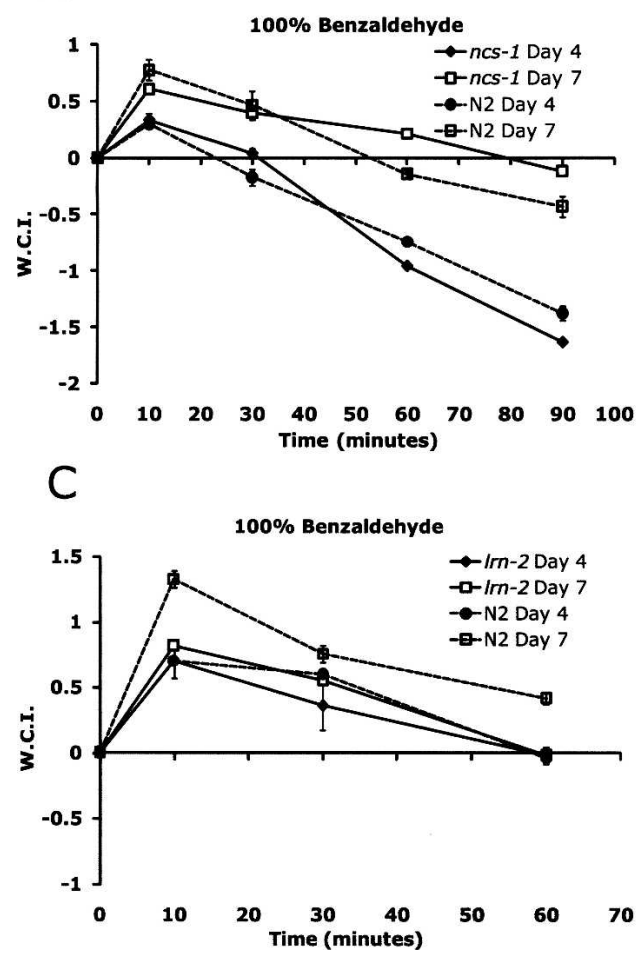

B

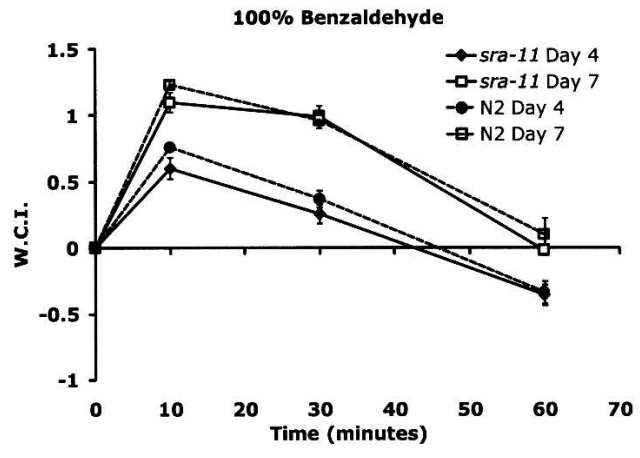

Figure 8. (A) Chemotaxis behavior ncs-1 mutants. Time course for chemotaxis to $100 \%$ benzaldehyde by day 4 vs. day 7 animals for N2 (dashed line) and $n c s-1$ mutants (solid lines). $n=3$ plates per data point for all data points. $(B)$ Chemotaxis behavior sra- 11 mutants. Time course for chemotaxis to $100 \%$ benzaldehyde by day 4 vs. day 7 animals for N2 (dashed line) and sra-11 mutants (solid lines). $n=3$ plates per data point for all data points. (C) Chemotaxis behavior Irn-2 mutants. Time course for chemotaxis to $100 \%$ benzaldehyde by day 4 vs. day 7 animals for N2 (dashed line) and Irn-2 mutants (solid lines). $n=3$ plates per data point for all data points.

worms. In contrast, there was a significant age $\times$ time interaction $\left(F_{(2,12)}=36.51, P<0.05\right)$ in control N2 worms. Post-hoc Bonferroni $t$-tests showed that day 7 animals had a higher chemotaxis index than day 4 animals at all time points for N2 animals $(P<0.05)$, but there was no difference in chemotaxis index between ages at all time points for $l r n-2$ animals $(P>0.05)$.

While the insulin pathway is also implicated in associative learning (Murakami et al. 2005; Tomioka et al. 2006), it may not be required in this form of learning, because a short-term odorfood learning assay (1-h pairing of benzaldehyde and food) failed to reveal any deficit in ins-1 (an antagonist for daf-2) animals (A. Lin and D. van der Kooy, unpubl.).

Together, these results suggest that the learned ageassociated increase in benzaldehyde attraction is mediated by a pathway involving $l r n-2$, but different from the pathways known for salt-food learning (ncs-1) and olfactory imprinting (sra-11).

\section{Discussion}

One might suggest that the age-related increase in chemotaxis toward benzaldehyde is a result of an aging-associated deficit in avoidance behavior, since avoidance behaviors are expressed only at high odorant concentrations, at which increased benzaldehyde chemotaxis is seen. However, three pieces of evidence refute this hypothesis and suggest instead that the stronger olfactory attraction is due to an enhanced attractive mechanism specifically at high odorant concentrations. First, odr-3 mutant animals that lack the attractive mechanism, and thus only avoid the odorant, showed no age-associated change in chemotaxis behavior. Second, no age-associated change in chemotaxis behavior was observed with an odorant that only elicits an avoid- ance response (octanol). Third, aged animals showed quicker and greater attraction to $1 \%$ benzaldehyde, a concentration that only elicits attraction and no avoidance. Together, these findings suggest that an early effect of aging is an enhancement in expression of attractive behavior to high concentrations of volatile odorants.

As with other sensory modalities, age-related changes in olfactory function are a result of complex interactions between genetically predisposed and experience-dependent changes. Histology of human and rodent epithelia and olfactory bulb reveal striking differences between young and old animals (Mulvaney 1971; Naessen 1971; Hinds and McNelly 1981). While changes in the structure of the sensory apparatus with age can result in change in olfactory function in humans and rodents, this is not a major cause for the change in the olfactory-mediated behavior that we observed in aged $C$. elegans. In advanced aged animals (day 18), sensory dendrites of chemosensory neurons were observed and cilia structures were normal (D. Hall, pers. comm.). While it has been reported that the cuticle of the worms thickens as age increases (Herndon et al. 2002), the passageway leading from the surface into the amphid pocket is still open in day 18 animals (D. Hall, pers. comm.). This suggests that the thickening does not block the channel for odorant molecules to enter and bind to their receptors on the olfactory sensory neuron dendrites. Indeed, we performed a DiI filling experiment and confirmed that the route to the amphid pocket is passable for dye molecules in both day 4 and day 7 animals (not depicted). Therefore, it is unlikely that the quicker and prolonged attraction to benzaldehyde and diacetyl is due to a morphological change in the sensory apparatus. 
Moreover, the results further suggest that the increase in attraction to benzaldehyde in aged animals is experience dependent. Specifically, it is dependent on the exposure to sensory cues from the bacterial food source the animals receive during their growth. There are two possible explanations for the nature of the experience dependence. One explanation is that the presence of odor cues released from $E$. coli has reshaped the behavior of the animals by a process known as olfactory imprinting. This is unlikely because olfactory imprinting occurs in a critical window that coincides with the first larval stage (Remy and Hobert 2005), long before the adult stages that are the period of interest in the present study. A second and more likely explanation is that by virtue of feeding on the $E$. coli for three more days, the day 7 animals received longer pairing between the presence of odorants (metabolites coming off from $E$. coli) and the presence of appetitive food than the day 4 animals, and hence, increased their attraction to the odorants. Indeed, worms were shown to be able to associate an olfactory cue with the presence of food in the adult stage (Nuttley et al. 2002). We explicitly tested this associative learning hypothesis by removing the olfactory cues (such as benzaldehyde and diacetyl) that predict the presence of food starting at the adult stage, and this manipulation abolished the increased attraction in aged animals. Worms were grown on nonbacterial food sources that did not release the bacterial metabolites benzaldehyde and diacetyl. Aged worms grown in this way, in which the contingency between benzaldehyde and appetitive food is lost, showed no greater attraction to benzaldehyde compared with young adult worms. While it is possible that the lack of benzaldehyde attraction when given axenic media from day 4 to day 7 could be partly due to extinction of foodbenzaldehyde pairing, this would require a second assumption that there is an intrinsic, unexplained enhanced attraction in day 7 animals. Moreover, reinitiating the odor-food association by returning worms to $E$. coli or supplementing the axenic media with benzaldehyde recovered the attraction. Thus, we conclude that longer associative learning between the presence of benzaldehyde (conditioned stimulus) and food (unconditioned stimulus) can explain the greater benzaldehyde attraction in aged worms. While genes that direct cell specification and cilia morphology are important for shaping worm olfactory behaviors during development (Coburn and Bargmann 1996; Roayaie et al. 1998; Lanjuin et al. 2003), the present data show that certain olfactory-mediated behaviors remain plastic throughout the adult stages of worm life. The worms are constantly modifying their behaviors based on the contingencies between sensory stimuli and appetitive cues present in the environment.

The results suggest that the increase in attraction to odorants is specific only to high odorant concentrations. Previous studies clearly suggested the presence of distinct olfactory signaltransduction mechanisms for different odorant concentrations. In addition to the identified high-affinity odr-10 diacetyl receptor on AWA, there is a low-affinity diacetyl receptor on AWC that mediates responses to high concentrations (100\%) of diacetyl and 2,3-pentanedione (Chou et al. 1996). rgs-3 mutants also show deficits in response to only high concentrations of stimuli such as isoamyl alcohol, another odorant sensed by AWC (Ferkey et al. 2007). Finally, pre-exposure to butanone and food also enhances chemotaxis to butanone at high concentrations, but not low concentrations (Torayama et al. 2007). The present data further supports the presence of separate high-affinity and lowaffinity chemotaxis pathways for benzaldehyde and suggests that the low-affinity pathway may be recruited by many of the odorants that worms detect. odr-3 may be the downstream G-protein in the transduction of this low-affinity pathway, since odr-3 mutants were deficient in the enhanced attraction to high concentrations of benzaldehyde in day 7 worms. Alternatively, odr-3 may be the downstream signaling protein for the olfactory cue after the association is formed, or it may play a role in the integration of sensory cues. While it is not clear what concentration of benzaldehyde is released from E. coli, it is possible that with $3 \mathrm{~d}$ of exposure, animals can form an appetitive association between the presence of food in the environment and the presence of even low concentrations of odorants. We propose that the associative learning between benzaldehyde released from the bacterial food source and the presence of appetitive food itself selectively facilitates the low-affinity chemotaxic pathway (which sense high concentrations of benzaldehyde) at or downstream from the olfactory receptor, but not the high-affinity chemotaxic pathway.

One might speculate that there would be a change in olfactory adaptation if there is a change in the low-affinity olfactory receptor. Day 7 animals appear to adapt at a slightly faster rate compared with day 4 animals, although there is no difference in their level of adaptation after an hour of exposure (Fig. 2C). The quicker learning may be due to an increased processing of high-concentration odorants (animals were adapted with 100\% benzaldehyde) due to an up-regulation of the low-affinity olfactory receptor or its downstream pathway as a result of selective modification by the associative learning. However, it is not clear whether olfactory adaptation is a phenomenon at the receptor level. While it has been shown that the cGMP-dependent protein kinase egl-4 in the primary sensory neuron AWC is important for adaptation (L'Etoile et al. 2002), another study showed that the downstream interneuron AIY controls olfactory adaptation via the Ras-MAPK pathway (Hirotsu and Iino 2005), suggesting olfactory adaptation may be mediated by a mechanism downstream from both the olfactory receptor level and primary sensory neuron. Furthermore, a longer period of adaptation $(2 \mathrm{~h})$ results in a negative chemotaxis index, which suggests that animals avoid, rather than lose sensitivity to the odorant after adaptation. Lastly, the presence of food or serotonin during training can block olfactory adaptation (Nuttley et al. 2002). All of these results suggest that olfactory adaptation is likely an associative learning phenomenon involving both primary sensory neuron and interneuron, rather than an unconditioned learning phenomenon. Therefore, a change at the olfactory receptor level may not contribute to a change in olfactory adaptation.

In C. elegans, tph-1 is expressed in serotonergic neurons including the two food sensing neurons-ADF and NSM (Sze et al. 2000), whereas mod-1 is expressed in interneurons including major targets of the AWC neurons-AIY and AIZ (Ranganathan et al. 2000). Both tph-1 and mod-1 were shown to mediate foodenhanced locomotor slowing and olfactory learning (Sawin et al. 2000; Zhang et al. 2005). Our results show that mutations in either tph-1 or mod-1 cause defects in age-enhanced attraction to benzaldehyde, which is a form of odor-food associative learning supported by our results, and suggest that serotonin may act on the serotonin-gated chloride channel to mediate this form of odor-food associative learning. Rescue experiments from previous studies show that serotonin released from different neurons plays a more prominent function in either food-related olfactory learning or food-modulated locomotion, depending on which neuron it is released from (Zhang et al. 2005). Thus, in the enhanced attraction we observed, serotonin may function as either a food signal or a mediator for associative learning, or both. Our results also show that $l r n-2$ (Fig. 8C) is required for the ageenhanced attraction to benzaldehyde, while ncs-1 (Fig. 8A) and sra-11 (Fig. 8B) are not required. These results argue that pathways for food-associative learning are distinct for particular sensory cue modalities. In this case, the pathway involved in the learned increased attraction to an olfactory stimulus differs from the pathway involved in temperature-food learning mediated 
by ncs-1 (Gomez et al. 2001). They also support the idea that different genes may be involved in learning in different specific stages of an animal's life cycle. Specifically, the sra-11 gene (involved in larval olfactory imprinting) (Remy and Hobert 2005) is not required for the form of adult olfactory learning in our paradigm. Altogether, these results further support our hypothesis that the enhanced attraction in aged animals is learned, and this learned increase in attraction to high concentrations of odors is mediated by the $l r n-2$ pathway.

There are many tastes and smells released by bacteria, the worm's food. It has been found that hundreds of metabolites are present in E. coli (Styczynski et al. 2007). However, these metabolites, including benzaldehyde and diacetyl, are abundant in nature. If the association between low concentrations of bacterialrelease metabolites and the presence of bacterial food also facilitates high-affinity attraction, then worms would approach these chemicals from considerable distances, even if the chemicals were not released by a food source. Hence, not only would it be energetically costly for worms to cover long distances, but they might also be exposed to potential predators, parasites, and toxic environments. Thus, the facilitation of a high-affinity chemoattractive pathway alone may reduce the worms' exposure to harmful stimuli and confer an evolutionary advantage over a learned generalization to low concentrations of odorants.

In conclusion, this study provides a novel and sensitive approach for identifying early physiological changes in aging $C$. elegans. It reveals that aging $C$. elegans develop an enhanced attraction to high concentrations of attractive volatile odorants compared with young adult worms. This enhancement is not caused by changes in locomotory rate and is specific to only high concentrations of odorants. We suggest that in addition to the two independent high-affinity chemoattractive mechanisms for benzaldehyde and diacetyl, there may be a separate, single, lowaffinity chemoattractive mechanism for both. Moreover, the enhanced attraction to high odorant concentrations is serotonin and experience dependent, and may be accounted for by a lifelong associative learning process that the worms experience between the odorants released by $E$. coli and the presence of the appetitive $E$. coli food source itself.

\section{Acknowledgments}

Thanks to Susan Runciman, Crystal Pegg, and Prateek Goyal for their excellent technical assistance and the Caenorhabditis Genetics Center at the University of Minnesota for the CX 2205 odr-3 (n2150), GR1321 tph-1 (mg280), MT9668 mod-1 (ok103), XA406 ncs-1 (qa406), and RB816 sra-11 (ok630) strains. This work was supported by the Natural Sciences and Engineering Research Council of Canada (NSERC).

\section{References}

Bargmann, C.I., Hartwieg, E., and Horvitz, H.R. 1993. Odorant-selective genes and neurons mediate olfaction in C. elegans. Cell 74: 515-527.

Bernhard, N. and van der Kooy, D. 2000. A behavioral and genetic dissection of two forms of olfactory plasticity in Caenorhabditis elegans: Adaptation and habituation. Learn. Mem. 7: 199-212.

Boehm, M. and Slack, F. 2005. A developmental timing microRNA and its target regulate life span in C. elegans. Science 310: 1954-1957.

Chao, M.Y., Komatsu, H., Fukuto, H.S., Dionne, H.M., and Hart, A.C. 2004. Feeding status and serotonin rapidly and reversibly modulate a Caenorhabditis elegans chemosensory circuit. Proc. Natl. Acad. Sci. 101: 15512-15517.

Chitwood, D.J., Lusby, W.R., Thompson, M.J., Kochansky, J.P., and Howarth, O.W. 1995. The glycosylceramides of the nematode Caenorhabditis elegans contain an unusual, branched-chain sphingoid base. Lipids 30: 567-573.

Chou, J.H., Troemel, E.R., Sengupta, P., Colbert, L., Tong, L., Tobin, D.M., Roayaie, K., Crump, J.G., Dwyer, N.D., and Bargmann, C.I. 1996. Olfactory recognition and discrimination in Caenorhabditis elegans. Cold Spring Harb. Symp. Quant. Biol. 61: 157-164.
Chow, D.K., Glenn, C.F., Johnston, J.L., Goldberg, I.G., and Wolkow, C.A. 2006. Sarcopenia in the Caenorhabditis elegans pharynx correlates with muscle contraction rate over life span. Exp. Gerontol. 41: $252-260$.

Coburn, C.M. and Bargmann, C.I. 1996. A putative cyclic nucleotide-gated channel is required for sensory development and function in C. elegans. Neuron 17: 695-706.

Doty, R.L., Shaman, P., Applebaum, S.L., Giberson, R., Siksorski, L., and Rosenberg, L. 1984. Smell identification ability: Changes with age. Science 226: 1441-1443.

Evason, K., Huang, C., Yamben, I., Covey, D.F., and Kornfeld, K. 2005. Anticonvulsant medications extend worm life span. Science 307: 258-262.

Ferkey, D.M., Hyde, R., Haspel, G., Dionne, H.M., Hess, H.A., Suzuki, H., Schafer, W.R., Koelle, M.R., and Hart, A.C. 2007. C. elegans G protein regulator RGS-3 controls sensitivity to sensory stimuli. Neuron 53: $39-52$.

Fukuto, H.S., Ferkey, D.M., Apicella, A.J., Lans, H., Sharmeen, T., Chen, W., Lefkowitz, R.J., Jansen, G., Schafer, W.R., and Hart, A.C. 2004. G protein-coupled receptor kinase function is essential for chemosensation in C. elegans. Neuron 42: 581-593.

Gerstbrein, B., Stamatas, G., Kollias, N., and Driscoll, M. 2005. In vivo spectrofluorimetry reveals endogenous biomarkers that report health span and dietary restriction in Caenorhabditis elegans. Aging Cell 4: $127-137$.

Glenn, C.F., Chow, D.K., David, L., Cooke, C.A., Gami, M.S., Iser, W.B., Hanselman, K.B., Goldberg, I.G., and Wolkow, C.A. 2004. Behavioral deficits during early stages of aging in Caenorhabditis elegans result from locomotory deficits possibly linked to muscle frailty. $J$. Gerontol. A Biol. Sci. Med. Sci. 59: 1251-1260.

Gomez, M., De Castro, E., Guarin, E., Sasakura, H., Kuhara, A., Mori, I. Bartfai, T., Bargmann, C.I., and Nef, P. 2001. $\mathrm{Ca}^{2+}$ signaling via the neuronal calcium sensor-1 regulates associative learning and memory in C. elegans. Neuron 30: 241-248.

Herndon, L.A., Schmeissner, P.J., Dudaronek, J.M., Brown, P.A., Listner, K.M., Sakano, Y., Paupard, M.C., Hall, D.H., and Driscoll, M. 2002. Stochastic and genetic factors influence tissue-specific decline in ageing C. elegans. Nature 419: 808-814.

Hinds, J.W. and McNelly, N.A. 1981. Aging in the rat olfactory system: Correlation of changes in the olfactory epithelium and olfactory bulb. J. Comp. Neurol. 203: 441-453.

Hirotsu, T. and Iino, Y. 2005. Neural circuit-dependent odor adaptation in C. elegans is regulated by the Ras-MAPK pathway. Genes Cells 10: $517-530$.

Hosono, R. 1978. Age dependent changes in the behavior of Caenorhabditis elegans on attraction to Escherichia coli. Exp. Gerontol. 13: $31-36$.

Lanjuin, A., VanHoven, M.K., Bargmann, C.I., Thompson, J.K., and Sengupta, P. 2003. Otx/otd homeobox genes specify distinct sensory neuron identities in C. elegans. Dev. Cell 5: 621-633.

L'Etoile, N.D., Coburn, C.M., Eastham, J., Kistler, A., Gallegos, G., and Bargmann, C.I. 2002. The cyclic GMP-dependent protein kinase EGL-4 regulates olfactory adaptation in C. elegans. Neuron 36: $1079-1089$.

Morrison, G.E., Wen, J.Y., Runciman, S., and van der Kooy, D. 1999. Olfactory associative learning in Caenorhabditis elegans is impaired in lrn-1 and lrn-2 mutants. Behav. Neurosci. 113: $358-367$.

Mulvaney, B.D. 1971. Chemography of lysosome-like structures in olfactory epithelium. J. Cell Biol. 51: 568-575.

Murakami, H., Bessinger, K., Hellmann, J., and Murakami, S. 2005. Aging-dependent and -independent modulation of associative learning behavior by insulin/insulin-like growth factor-1 signal in Caenorhabditis elegans. J. Neurosci. 25: 10894-10904.

Naessen, R. 1971. An enquiry on the morphological characteristics and possible changes with age in the olfactory region of man. Acta Otolaryngol. 71: 49-62.

Nierop Groot, M.N. and de Bont, J.A.M. 1998. Conversion of phenylalanine to benzaldehyde initiated by an aminotransferase in Lactobacillus plantarum. Appl. Environ. Microbiol. 64: 3009-3013.

Nuttley, W.M., Harbinder, S., and van der Kooy, D. 2001. Regulation of distinct attractive and aversive mechanisms mediating benzaldehyde chemotaxis in Caenorhabditis elegans. Learn. Mem. 8: 170-181.

Nuttley, W.M., Atkinson-Leadbeater, K.P., and van der Kooy, D. 2002. Serotonin mediates food-odor associative learning in the nematode Caenorhabditis elegans. Proc. Natl. Acad. Sci. 99: 12449-12454.

Ranganathan, R., Cannon, S.C., and Horvitz, H.R. 2000. MOD-1 is a serotonin-gated chloride channel that modulates locomotory behaviour in C. elegans. Nature 408: 470-475.

Remy, J.J. and Hobert, O. 2005. An interneuronal chemoreceptor required for olfactory imprinting in C. elegans. Science 309: 787-790.

Roayaie, K., Crump, J.G., Sagasti, A., and Bargmann, C.I. 1998. The G $\alpha$ protein ODR-3 mediates olfactory and nociceptive function and 
controls cilium morphogenesis in C. elegans olfactory neurons. Neuron 20: 55-67.

Sawin, E.R., Ranganathan, R., and Horvitz, H.R. 2000. C. elegans locomotory rate is modulated by the environment through a dopaminergic pathway and by experience through a serotonergic pathway. Neuron 26: 619-631.

Sengupta, P., Chou, J.H., and Bargmann, C.I. 1996. odr-10 encodes a seven transmembrane domain olfactory receptor required for responses to the odorant diacetyl. Cell 84: 899-909.

Styczynski, M.P., Moxley, J.F., Tong, L.V., Walther, J.L., Jensen, K.L., and Stephanopoulos, G.N. 2007. Systematic identification of conserved metabolites in GC/MS data for metabolomics and biomarker discovery. Anal. Chem. 79: 966-973.

Sze, J.Y., Victor, M., Loer, C., Shi, Y., and Ruvkun, G. 2000. Food and metabolic signalling defects in a Caenorhabditis elegans serotonin-synthesis mutant. Nature 403: 560-564.

Tomioka, M., Adachi, T., Suzuki, H., Kunitomo, H., Schafer, W.R., and Iino, Y. 2006. The insulin/PI 3-kinase pathway regulates salt chemotaxis learning in Caenorhabditis elegans. Neuron 51: 613-625. Torayama, I., Ishihara, T., and Katsura, I. 2007. Caenorhabditis elegans integrates the signals of butanone and food to enhance chemotaxis to butanone. J. Neurosci. 27: 741-750.

Troemel, E.R., Chou, J.H., Dwyer, N.D., Colbert, H.A., and Bargmann, C.I. 1995. Divergent seven transmembrane receptors are candidate chemosensory receptors in C. elegans. Cell 83: 207-218.

Wen, J.Y.M., Kumar, N., Morrison, G., Rambaldini, G., Runciman, S., Rousseau, J., and van der Kooy, D. 1997. Mutations that prevent associative learning in C. elegans. Behav. Neurosci. 111: 1-15.

Zhang, Y., Chou, J.H., Bradley, J., Bargmann, C.I., and Zinn, K. 1997. The Caenorhabditis elegans seven-transmembrane protein ODR-10 functions as an odorant receptor in mammalian cells. Proc. Natl. Acad. Sci. 94: 12162-12167.

Zhang, Y., Lu, H., and Bargmann, C.I. 2005. Pathogenic bacteria induce aversive olfactory learning in Caenorhabditis elegans. Nature 438: $179-184$

Received August 11, 2008; accepted in revised form August 14, 2008. 


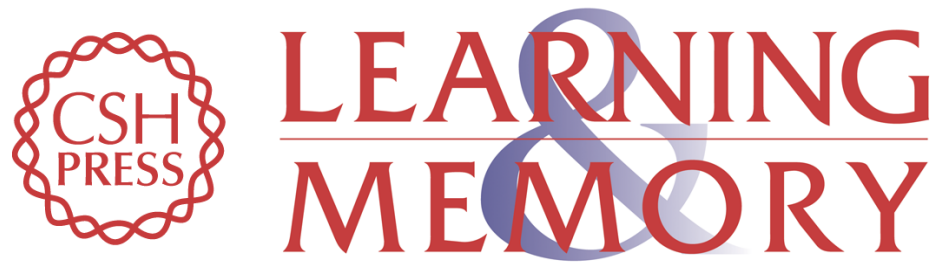

\section{Serotonin mediates a learned increase in attraction to high concentrations of benzaldehyde in aged $C$. elegans}

David Tsui and Derek van der Kooy

Learn. Mem. 2008, 15:

Access the most recent version at doi:10.1101//m.1188208

References This article cites 41 articles, 14 of which can be accessed free at: http://learnmem.cshlp.org/content/15/11/844.full.html\#ref-list-1

License

Email Alerting Receive free email alerts when new articles cite this article - sign up in the box at the Service top right corner of the article or click here. 